\title{
Photothermal radiometry for the estimation of the thickness of a protective chromium layer deposited on a steel substrate
}

\author{
J.L. Bodnar ${ }^{1, a}$, O. Faugeroux ${ }^{2}$ and B. Claudet ${ }^{2}$ \\ 1 GRESPI/ECATHERM, Université de Reims Champagne Ardenne, BP 1039, 51687 Reims, France \\ 2 PROMES, Université de Perpignan Via Domitia, 52 Avenue Paul Alduy, 66860 Perpignan, France
}

Received 7 January 2014, Accepted 15 May 2014

\begin{abstract}
The measurement of the thickness of a coating layer is a traditional industrial problem. Many methods of measurement are already classically used for that purpose. However, the majority of these methods require either contact with the sample to be studied, or its destruction. These constraints limit their applicability. Photothermal radiometry is already largely used in the field of thermophysical properties measurement and for the detection and characterization of defects. This method does not impose the constraints mentioned above. It could thus be a complementary technique of the classical ones. The purpose of this work is then to approach the possibilities of this photothermal method, implemented under pulse excitation and front face analysis, for the measurement of the coating thickness. Using theoretical and experimental studies, we show that the method allows a good estimation of the thickness of chromium deposits on a steel substrate, at distance, without contact and in a non-destructive way.
\end{abstract}

Key words: Non-destructive testing / photothermal radiometry / metals / thickness / measurement

\section{Introduction}

Measurement of the thickness of a coating layer is a common industrial problem. Many classical measurement methods already exist. Some of them are destructive, some are non-destructive. To mention just a few, one can cite measurement by magnetic attraction, by magnetic induction, by Foucault current, by coulometry, by optical cutting microscopy or beta-ray backscattering $[1,2]$. However, these methods are not universal and of course show some limitations. For instance, destructive methods do not allow the re-use of the sample after testing. As for non-destructive methods, most of them require contact between the studied sample and the measurement tool. New methods have therefore to be developed to achieve remote contact less non-destructive thickness measurement.

Among such new control methods, there is photothermal radiometry. Its principle is as follows: the sample under study is submitted to a light flux; its temperature locally increases; the variations of radiant emittance are recorded through an IR optical detection line. The photothermal signal obtained thus depends on the thermophysical properties of the studied material, so its characterization is possible [3-8]. This control method, working without contact and in a non-destructive way, seems

\footnotetext{
${ }^{a}$ Corresponding author: jl.bodnar@univ-reims.fr
}

promising for the measurement of the thickness of a coating. The present study aims to show the potentialities of photothermal radiometry in this field. This paper is divided in five parts. First, we explain the principle of front face impulse photothermal radiometry. Then, we present the characteristics of the photothermal signal recorded for a two-layer material (chromium coating on a steel substrate). Several methods of exploitation of this signal are explained. In a fourth step, the experimental set-up is described. Lastly, we show that our method allows a good estimation of the thickness for 6 different chromium coatings on steel substrate.

\section{Impulse photothermal radiometry}

Impulse photothermal radiometry is a non-destructive control method designed at the beginning of the 60s by Parker et al. [9]. Its success was immediate thanks to its simplicity and rapidity of implementation. It consists in lighting uniformly and for a very short time the surface of the sample to be characterized. The surface temperature increases. This temperature variation is linked to the thermal properties of the sample, so it is possible to characterize them by analyzing this variation. Initially, Parker et al. propose measuring the temperature variation with a thermocouple. This is embarrassing since there is 

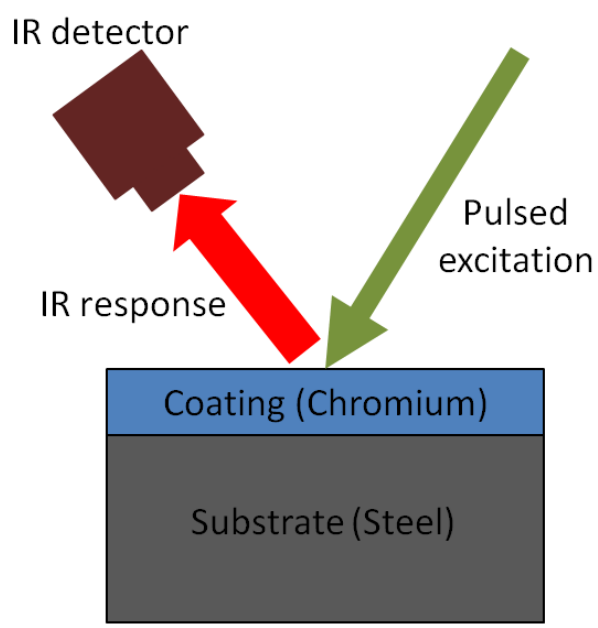

Fig. 1. Principle of the front face impulse photothermal radiometry.

contact between the sample and the measurement tool. Deem et al. [10] improve this NDT method to allow contact less analyses. They use a laser to excite the sample under study and an IR radiometer to measure the resultant temperature variations. To justify their approach, they show that in the case of a low thermal perturbation, the recorded IR photothermal signal is proportional to the surface temperature variation $\Delta T(t)$ of the sample:

$$
\operatorname{PTR}(t)=\mathrm{cte} \cdot \Delta T(t)
$$

This new approach enables many industrial applications to be envisaged. Many research projects are developed by scientists. Their results show that photothermal radiometry allows the study of all the parameters governing the temperature variation directly (diffusivity, effusivity...) or indirectly (presence of cracks, delaminations, structure of the sample...). Since a thickness variation may modify the temperature distribution, it seems a good idea to try to use photothermal radiometry to measure the thickness of a metallic coating. There are two different ways to implement impulse photothermal radiometry. The first consists in exciting the studied sample on one face and measuring the induced temperature variations on the opposite face. This method is known as "rear face analysis". Its main advantage is to increase strikingly the signal-tonoise ratio. But its drawback is that it is necessary to have access to the two faces of the sample. It is also slower than the second method, the "front face analysis". In this variant, the excitation and the temperature measurement are done on the same face. The signal is more affected by the noise but access to the rear face of the sample is not compulsory. Its rapidity makes it interesting for the industrial world. That is why we have chosen the "front face" method.

\section{Theoretical model developed for the study}

Before designing the experimental setup, we wanted to study theoretically the possibilities of the impulse photothermal method for the measurement of the thickness of a chromium coating. For that purpose, we modeled the photothermal experiment previously described. To solve the heat equation, we used the thermal quadrupoles formalism. Let us detail the hypotheses and the mode of resolution of the problem. First, we considered a two-layer material. The first layer is the steel substrate. The second one is the chromium coating. Given the conductive nature of these materials, we assumed that they were isotropic and opaque. The opacity of the coating layer allowed us to assume that the whole energy brought by the impulse excitation would be absorbed by the chromium coating surface. Moreover, the quickness of the energy deposit allowed us to neglect the convective losses on the different faces of the sample. For reasons of simplicity, we also assumed that the energy deposit was spatially homogeneous and temporarily similar to a Dirac function. We also assumed that there was perfect contact between coating and substrate and that the thermophysical properties were constant with regards to time and temperature. Lastly, the heat diffusion was assumed unidirectional. These hypotheses lead to the following matrix system:

$$
\begin{aligned}
{\left[\begin{array}{c}
\theta_{\text {front }}(p) \\
\Phi_{\text {front }}(p)
\end{array}\right]=} & {\left[\begin{array}{ll}
A_{\text {chromium }} & B_{\text {chromium }} \\
C_{\text {chromium }} & D_{\text {chromium }}
\end{array}\right] } \\
& \times\left[\begin{array}{ll}
A_{\text {steel }} & B_{\text {steel }} \\
C_{\text {steel }} & D_{\text {steel }}
\end{array}\right]\left[\begin{array}{l}
\theta_{\text {rear }}(p) \\
\Phi_{\text {rear }}(p)
\end{array}\right]
\end{aligned}
$$

where:

$$
\begin{aligned}
& A=\operatorname{Cosh}\left(e \sqrt{\frac{p}{a}}\right), B=\frac{\operatorname{Sinh}\left(e \sqrt{\frac{p}{a}}\right)}{\lambda \sqrt{\frac{p}{a}}}, \\
& C=\lambda \sqrt{\frac{p}{a}} \operatorname{Sinh}\left(e \sqrt{\frac{p}{a}}\right), D=\operatorname{Cosh}\left(e \sqrt{\frac{p}{a}}\right) .
\end{aligned}
$$

Then, the temperature is expressed in Laplace space as follows:

$$
\theta_{\text {front }}(p)=\frac{A_{\text {chromium }} A_{\text {steel }}+B_{\text {chromium }} C_{\text {steel }}}{C_{\text {chromium }} A_{\text {steel }}+D_{\text {chromium }} C_{\text {steel }}} \Phi_{\text {front }}(p)
$$

The return in temporal space is done using a numerical inverse Laplace transform performed by Stefest's numerical method [12].

$$
T_{\text {front }}(t)=\ln (2) \sum_{i=1}^{N}\left[\eta_{i} \theta_{\text {front }}\left(\frac{i \ln (2)}{t}\right)\right]
$$

with $\eta_{1}=0.08 ; \eta_{2}=-32.08 ; \eta_{3}=1279 ; \eta_{4}=-15623.67$; $\eta_{5}=84244.17 ; \eta_{6}=-236957.51 ; \eta_{7}=375911.9 ; \eta_{8}=$ $-340071.69 ; \eta_{9}=164062.51 ; \eta_{10}=-32812.50$.

\section{Exploitation method}

Using the mathematical model presented previously, we performed different simulations to estimate the influence of a variation of the thickness of the chromium 


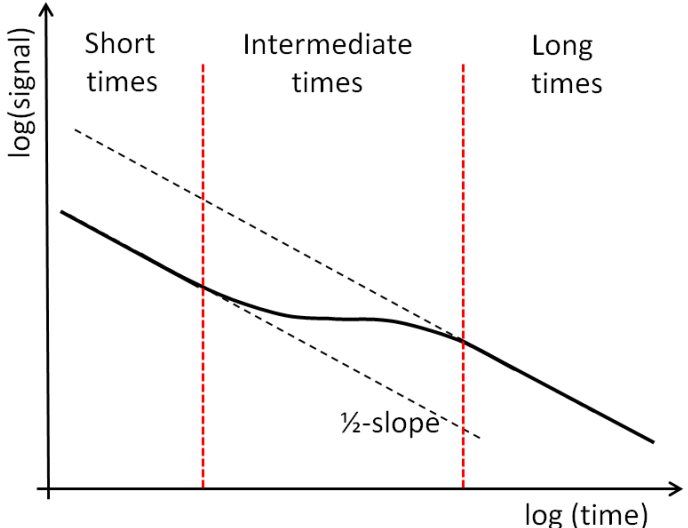

Fig. 2. Example of an impulse photothermal response for a two-layer material.

layer on the photothermal response. For these simulations, we considered the following thermal properties: for the chromium layer, a thermal conductivity of $70 \mathrm{~W} \cdot \mathrm{m}^{-1} \cdot \mathrm{K}^{-1}$, a thermal diffusivity of $2.5 \times 10^{-5} \mathrm{~m}^{2} . \mathrm{s}^{-1}$ and a thickness varying from $20 \mu \mathrm{m}$ to $200 \mu \mathrm{m}$, by steps of $10 \mu \mathrm{m}$. For the steel layer, we took a thermal conductivity of $38.2 \mathrm{~W} \cdot \mathrm{m}^{-1} \cdot \mathrm{K}^{-1}$, a thermal diffusivity of $1.1 \times 10^{-5} \mathrm{~m}^{2} \cdot \mathrm{s}^{-1}$ and a thickness of $2.5 \mathrm{~cm}$. These values correspond to those usually met in industry [13]. In figure 2, we present in logarithmic coordinates the photothermal response of a two-layer material. Three temporal zones clearly appear. The first zone corresponds to the short times. Figure 2 shows that in this zone, the photothermal response presents a $-1 / 2$ slope. Such a slope is characteristic of the response of a semi-infinite homogeneous material. In this configuration, the surface temperature is given by equation (5), which corresponds in logarithmic coordinates to a decreasing line of slope $-1 / 2$ [14]:

$$
T(t)=\frac{Q}{b \sqrt{\pi t}}
$$

This means that in the « short times $»$ zone, the thermal perturbation propagates only in the coating layer (Fig. 3). The third zone of the photothermal response shows another curve of slope $-1 / 2$. This zone corresponds to the long times, when the thermal perturbation propagates in the steel substrate (Fig. 3). Lastly, Figure 2 shows an intermediate zone, where the thermal perturbation is sensitive to the presence of the chromium/steel interface. It corresponds to the times when the perturbation crosses the coating/substrate interface. The temporal localization of this zone obviously depends on the thickness of the chromium layer. So it is in that zone that we should perform the estimation of that thickness.

At this stage of our study, we still had to determine the best way to exploit this intermediate zone of the photothermal signal. Several methods of exploitation were considered. The first one consisted in measuring the value of the amplitude of the photothermal signal at a given time. Figure 4 presents an example of a result obtained in that way. It corresponds to the analysis of the

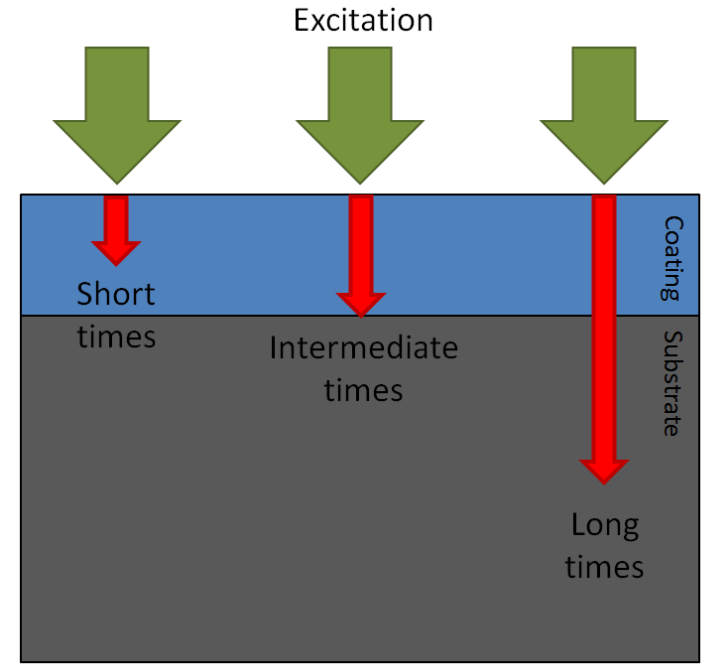

Fig. 3. Variation of the penetration depth of the thermal perturbation according to the analysis times.

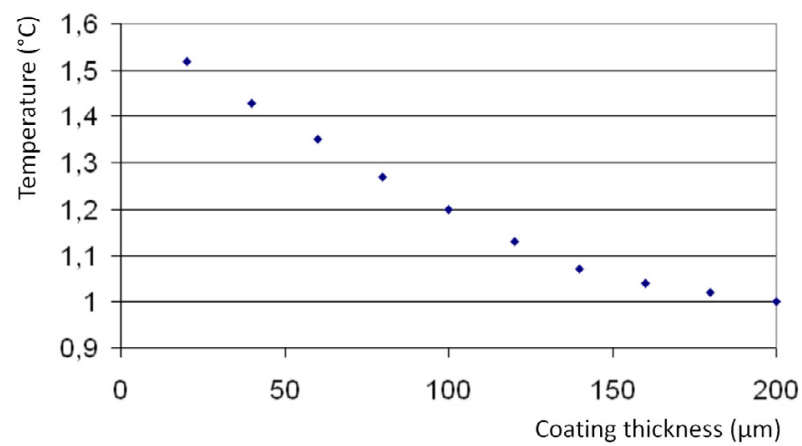

Fig. 4. Evolution of the photothermal signal with the chromium coating thickness, $0.1 \mathrm{~ms}$ after the end of the excitation.

photothermal signal recorded for a chromium-coated steel sample, $0.1 \mathrm{~ms}$ after the end of the excitation. It reveals a correlation between the value of the photothermal signal and the chromium thickness. So it shows the potential of this method to estimate this thickness. However, although this exploitation method is very simple, it requires a reproducible deposit of energy for all the samples under study. This condition is rather difficult to achieve experimentally. It is quite impossible to free ourselves from potential variations of the radiative properties of the studied samples or some fluctuations of the laser. So we chose to use an other method.

The second possible method to exploit the photothermal signal is an integral analysis. It consists in calculating the area included under the impulse response for a twolayer material (steel substrate with chromium coating) on the one hand and for an homogeneous material (steel substrate) on the other hand and then making the difference between the obtained results. Figure 5 shows an example of a result obtained in that way. There is clearly a correlation between this integral difference and the thickness of the chrome coating. Furthermore, compared with the previous one, this method has the advantage of taking 


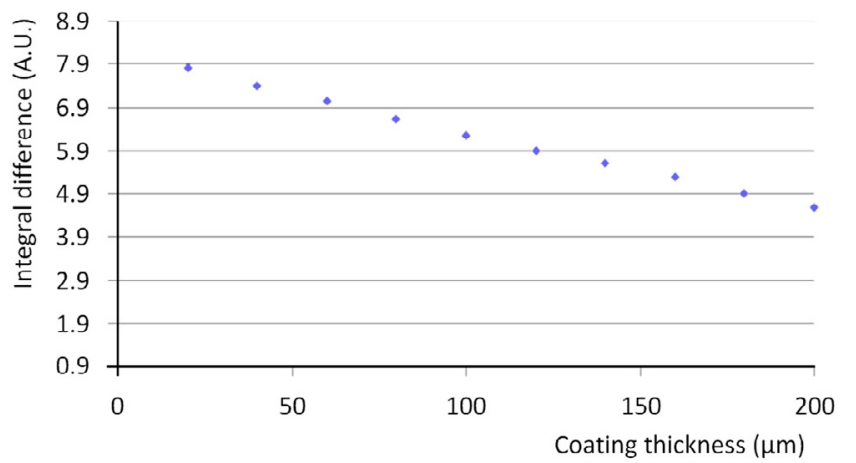

Fig. 5. Evolution of the integral difference with the chromium thicknes.

into account a large number of experimental points, thus improving the signal-to-noise ratio. In fact, it applies a low-pass filter to the photothermal signal, which by nature increases the signal-to-noise ratio. However, it also requires an experimental set-up that is stable from an energetic point of view. Since we did not know the real performances of our set-up, we preferred not to retain this method.

The third possible method is the no-follow time technique. It consists in locating the transition time between the "short-time" regime and the "intermediatetime" regime (Fig. 2) then correlating this characteristic time to the coating thickness. The main advantage of this method is that unlike the two previous ones, it uses a temporal measurement instead of an energetic one. It is thus potentially less sensitive to the inhomogeneities of the energy deposit (laser fluctuations or local variations of the radiative properties). Moreover, it allows a faster analysis since it only requires access to the short times and the beginning of the intermediate times of the impulse photothermal response. It is an advantage from an industrial point of view but, also at a theoretical level. It allows us to consider an one-dimensional propagation of heat and thus to use simpler mathematical models. It also allows a better understanding of the thermophysical phenomena involved during the photothermal experiment. For these reasons, this exploitation method has been chosen for our study. At this stage, we had to check the existence of a correlation between the no-follow time and the thickness of the chrome layer. In Figure 6, we plotted the theoretical photothermal responses calculated for a chrome thickness of $50 \mu \mathrm{m}, 100 \mu \mathrm{m}$ and $150 \mu \mathrm{m}$ respectively, as well as the "short-time" asymptotic behavior. One can clearly see that the photothermal responses leave this asymptotic behavior the sooner the chrome thickness is lower.

In Figure 7, we plotted the evolution of the no-follow time with the chromium thickness. It shows a monotonous increasing curve. So the existence of a correlation is proved. Moreover, it shows that for the chromium thicknesses we considered in our study, we will have to design an experimental set-up able to work at frequencies of about $100 \mathrm{kHz}$.

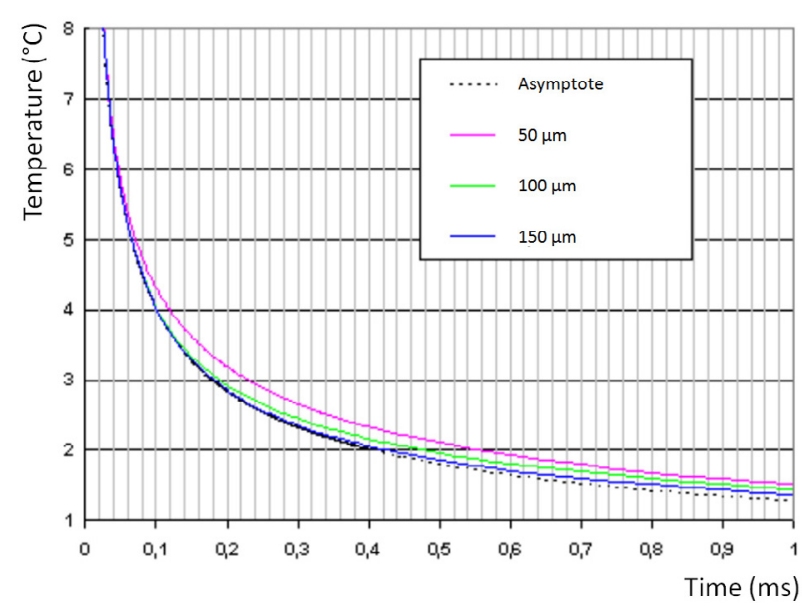

Fig. 6. Photothermal responses obtained for a chrome thickness of $50 \mu \mathrm{m}, 100 \mu \mathrm{m}$ and $150 \mu \mathrm{m}$, respectively.

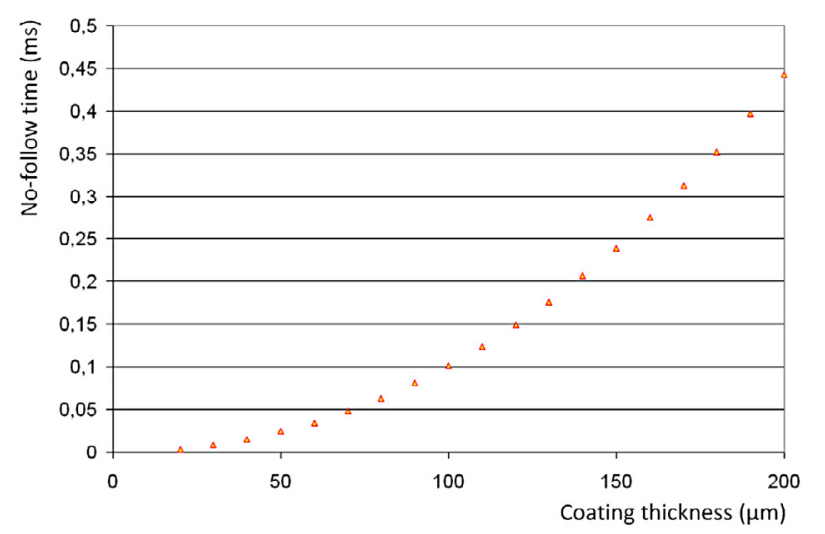

Fig. 7. Evolution of the no-follow time with the chrome thickness.

\section{Experimental set-up}

After that promising theoretical study, the following step was to design the experimental set-up. It consists of three parts: an optical excitation line, an IR detection line and the driving electronic and computer system (Fig. 8). The optical excitation line is comprised of a laser source, an attenuation optic and a shaping optic. The light source is a Nd:Yag pulsed laser. The impulse duration is about $6 \mathrm{~ns}$ and the available maximal energy is about $450 \mathrm{~mJ}$. The diameter of the exciting laser beam is close to $1 \mathrm{~cm}$. The attenuation optics consists of several treated glass blades. The shaping optics consists of a lens doublet. The IR detection line is comprised of a detector and a collecting optics. The IR detector is a quantum detector cooled by liquid nitrogen. With a time constant lower than a microsecond, it is suitable for our study. Its sensitive surface is about $1 \mathrm{~mm}^{2}$. The lit surface is much bigger, so we can assume the problem to be one-dimensional. The collecting optics consists of a ZnSe lens doublet coupled with a reflective hemisphere drilled in its center and placed on the sample to be analyzed. The hemisphere increases the apparent radiative properties of the surface of the studied 


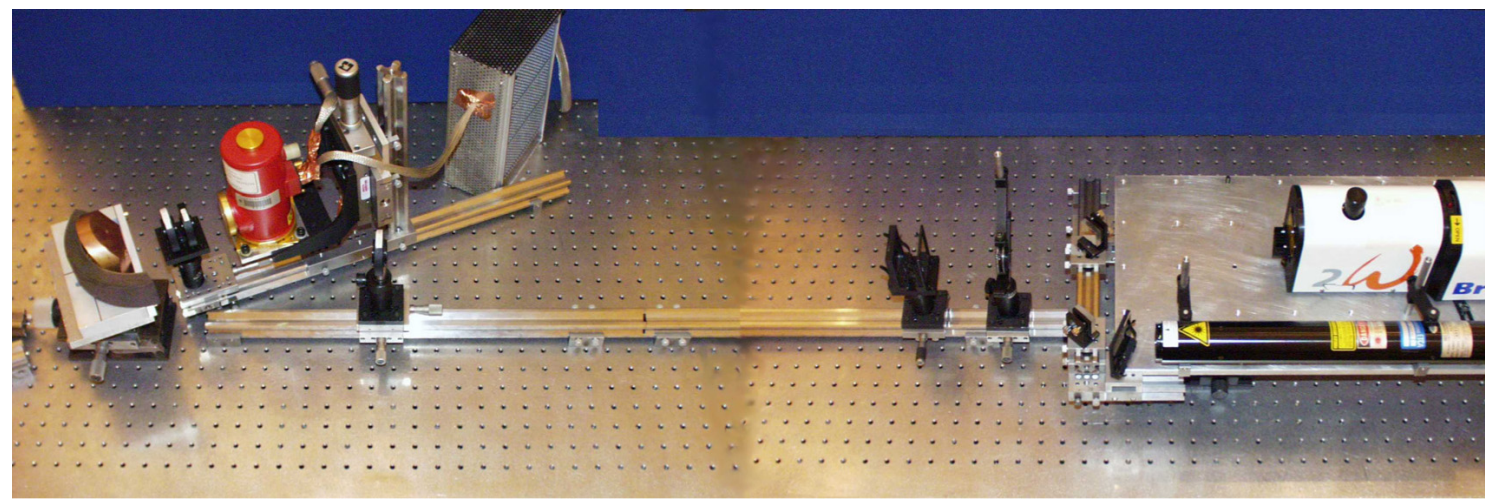

Fig. 8. Experimental set-up.

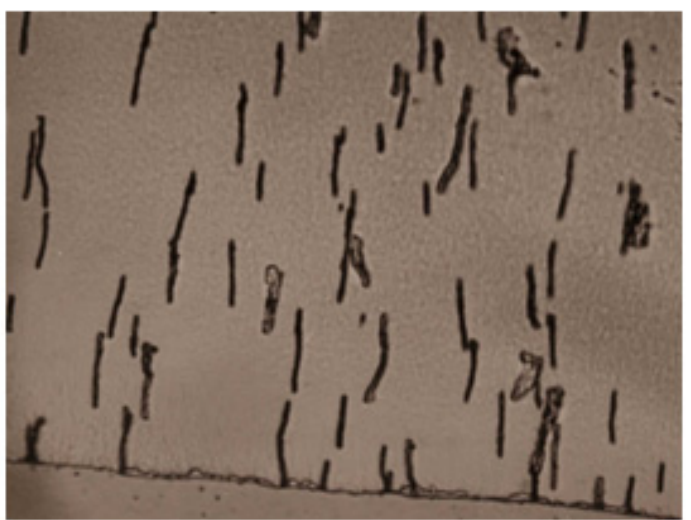

Fig. 9. Example of a chromium coating.

sample. The detector is connected to an amplifier and a digitalization unit. A computer drives the set-up.

\section{Experimental results}

\subsection{Samples under study}

We had 6 samples at our disposal. They were made of a layer of electrolytic chromium deposited on a steel substrate. Figure 9 shows a micrograph of the surface of one of these samples. The thicknesses of the chrome layer were measured by a scanning electron microscope. They are $130 \mu \mathrm{m}, 138 \mu \mathrm{m}, 130 \mu \mathrm{m}, 50 \mu \mathrm{m}, 58 \mu \mathrm{m}, 55 \mu \mathrm{m}$, respectively.

\subsection{Experimental results}

Each sample was analyzed with our experimental setup without a priori knowledge of its thickness. An example of the obtained results is shown in Figure 10. The response is a decreasing monotonous curve, divided into three parts. During the first twenty microseconds, the photothermal response varies appreciably in time and does not show the $-1 / 2$ slope theoretically expected. We impute this detection artifact to a non-linear response

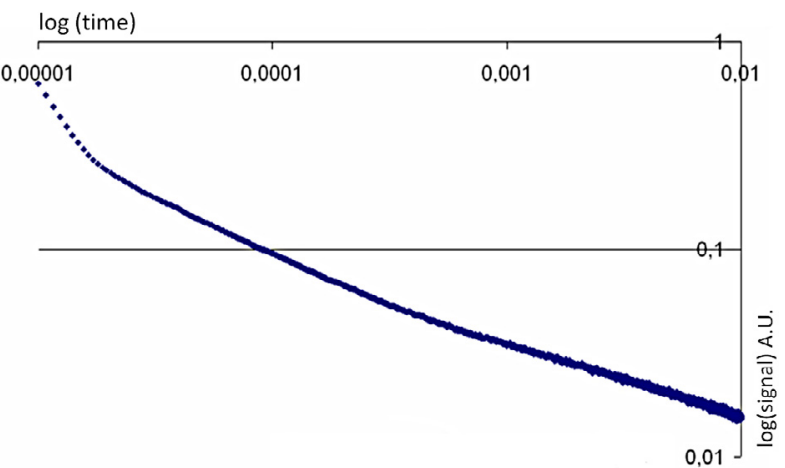

Fig. 10. Example of an experimental photothermal response.

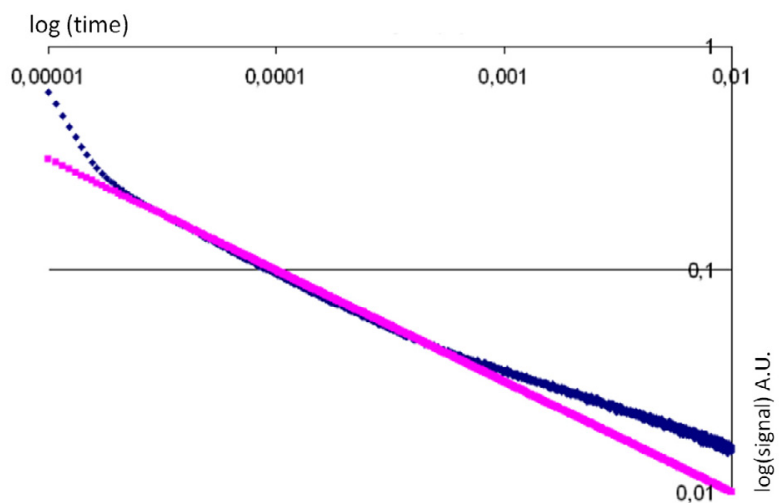

Fig. 11. Example of fitting between theory and experiment.

of the detector facing the intense radiation following the laser pulse. The second part of the photothermal response presents as expected a negative slope equal to $-1 / 2$. That interval corresponds to the propagation of the thermal perturbation in the coating layer. Then, and again as expected in theory, the curve shows a slope change, due to the propagation of the energy in the steel substrate.

In order to estimate the thickness of the chrome layer, we tried to determine the no-follow time. For that purpose, we fitted to the experimental signal a decreasing line of slope $-1 / 2$ (Fig. 11).

Then, we subtracted the two curves. The obtained result is presented in Figure 12. 


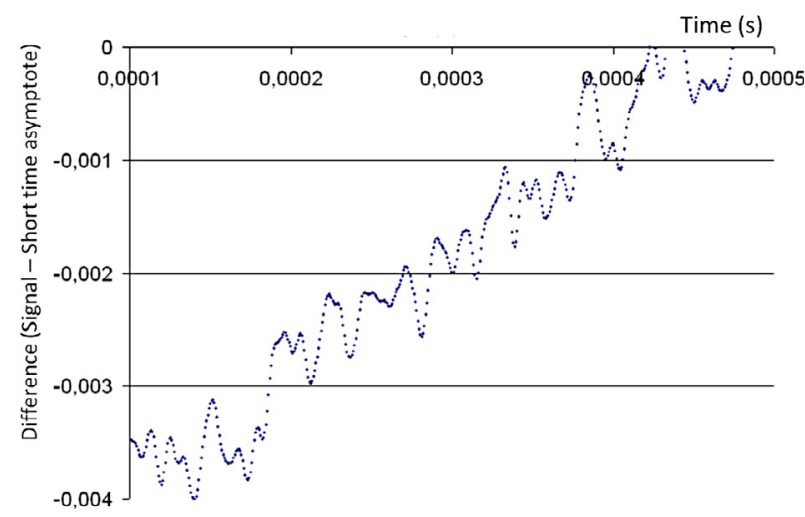

Fig. 12. Example of the difference between the photothermal signal and the short-time asymptot.

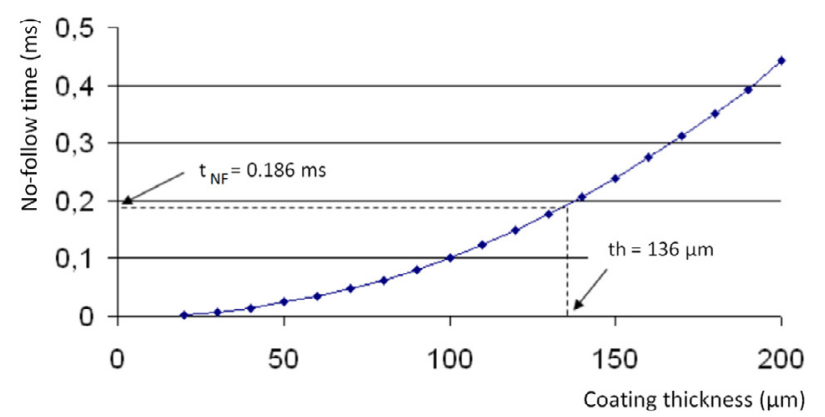

Fig. 13. Example of the estimation of the chromium thickness.

Table 1. Experimental results.

\begin{tabular}{lcccc}
\hline & td $(\mathrm{s})$ & $\begin{array}{c}\text { Estimated } \\
\text { thickness } \\
(\mu \mathrm{m})\end{array}$ & $\begin{array}{c}\text { Real } \\
\text { thickness } \\
(\mu \mathrm{m})\end{array}$ & $\begin{array}{c}\text { Relative } \\
\text { error } \\
(\%)\end{array}$ \\
\hline Sample 1 & $1.19 .10^{-4}$ & 109.6 & 130 & 15.7 \\
Sample 2 & $1.86 .10^{-4}$ & 136,5 & 138 & 1.1 \\
Sample 3 & $1.99 .10^{-4}$ & 140.6 & 130 & 8.2 \\
Sample 4 & $0.33 .10^{-4}$ & 54.6 & 50 & 9.2 \\
Sample 5 & $0.32 .10^{-4}$ & 54.5 & 58 & 6 \\
Sample 6 & $0.38 .10^{-4}$ & 58.4 & 55 & 6.2 \\
\hline
\end{tabular}

In a third step, we extracted from this difference the no-follow time we were looking for. In the example presented above, we estimated this time at $0.186 \mathrm{~ms}$. Finally, we used this value in Figure 7 to determine the thickness. The result is about $136 \mu \mathrm{m}$ (Fig. 13).

We proceeded in the same way for the five other samples and obtained the results presented in Table 1 . If we compare with the real values of the thickness, we can conclude that the front face impulse photothermal radiometry coupled with the determination of the characteristic no-follow time allows, in the six cases, a good estimation of the required parameter. That feasibility study shows that the photothermal method is well adapted to measure the thickness of a metallic coating.

\section{Conclusion}

This study aimed at approaching the possibilities of impulse photothermal radiometry for the estimation of the thickness of a chromium coating. The interest of the photothermal method is to potentially give access to an industrialisable non-destructive method working at distance and without contact. Using a mathematical modeling of the impulse photothermal experiment, we first showed that there was a correlation between the no-follow time and the thickness of the chromium layer. This characteristic time corresponds to the time when the thermal perturbation crosses the coating/substrate interface. The photothermal signal leaves its asymptotic behavior of slope $-1 / 2$ for a less sharp one. This change of slope is highly visible graphically, hence its utilization. We insisted on the fact that this exploitation method presented two other main interests. On the one hand, from an industrial point of view, it allows a fast analysis and so a potential productivity gain. On the other hand, from a theoretical point of view, simple one-dimensional models can be used. It facilitates the modeling of the experiment and the comprehension of the thermophysical phenomena involved. Then we presented the experimental set-up designed for the study. Finally, we studied six industrial samples previously characterized by scanning electron microscopy and showed that the photothermal method allowed an accurate estimation of the chrome coating thickness. These first results obtained for particular samples are very encouraging and should give access to an industrialisable remote contact less non-destructive method for measuring the thickness of a protective metallic layer. Now they should be confirmed and generalized. It would be interesting to study the influence on the estimated thickness of an uncertainty regarding the values of the thermophysical properties of the chromium layer as well as an imperfect contact between the substrate and the coating. Such studies are in progress.

\section{References}

[1] R. Levy, M. Saurat, Contrôle des revêtements en production industrielle, TI, 1985, fiche r 1680

[2] J. Dumont-Fillon, Mesure et contrôle; Contrôle non destructif, TI, 1996, fiche r 1400

[3] X. Maldague, Theory and practice of infrared technology for non destructive testing

[4] V.P. Vavilov, Non destructive testing handbook: ther$\mathrm{mal} /$ infrared testing

[5] Le Contrôle des matériaux par méthodes optiques infrarouge, Livre : Méthodes et Techniques Optiques pour l'Industrie, Collectif d'auteurs, dirigé par Paul Smigielski, ISBN : 978-2-918241-00-3, code EAN : 9782918241003, Société Française d'Optique, novembre 2009, pp. $232-296$

[6] J.C. Candoré, J.J. Bodnar, V. Detalle, P. Grossel, Nondestructive testing of works of art by stimulated infrared thermography, Eur. Phys. J. Appl. Phys. 57 (2012) 21002

[7] J.C. Candoré, J.L. Bodnar, V. Detalle, P. Grossel, Characterization of defects situated in a fresco by 
stimulated infrared thermography, Eur. Phys. J. Appl. Phys. 57 (2012) 11002

[8] J.L. Bodnar, J.C. Candoré, J.L. Nicolas, G. Szatanik, V. Detalle, J.M. Vallet, Stimulated infrared thermography applied to help restoring mural paintings, NDT E Int. 49 (2012) 40-46

[9] W.J. Parker, R.J. Jenkins, C.P. Butler, G.L. Abbot, Flash method of determining thermal diffusivity, heat capacity,and thermal conductivity, J. Appl. Phys. 32 (1961) $1679-1684$

[10] H.W. Deem, W.D. Wood, Rev. Sci. Instrum. 33 (1962) 1107-1109
[11] D. Maillet, S. André, J.C. Batsale, A. Degiovanni, C. Moyne, Thermal quadrupoles. Solving the heat equation through integral transforms, Wiley, 2000

[12] H. Stefest, « Remarks on algorithm $368 »$, A.C.M. Commun. 13 (1970) 624

[13] O. Faugeroux, «Caractérisation thermophysique de revêtement de protection thermomécanique par méthode photothermique impulsionnelle », Ph.D. thesis, Université de Perpignan, Décembre 2001

[14] J. Hladik, Métrologie des propriétés thermophysiques des matériaux, Masson, 1990 